\title{
Cardiac arrest during percutaneous coronary intervention in a patient 'resistant' to clopidogrel - successful 50-minute mechanical chest compression
}

\author{
Marcin Protasiewicz ${ }^{1}$, Pawel Szymkiewicz ${ }^{1}$, Krzysztof Sciborski $^{1}$, Alina Orda ${ }^{1}$, Bozena Karolko $^{1}$, Anna Jonkisz ${ }^{2}$, \\ Arleta Lebioda², Andrzej Mysiak ${ }^{1}$
}

${ }^{1}$ Department of Cardiology, Medical University of Wroclaw, Poland

${ }^{2}$ Department of Forensic Medicine, Medical University of Wroclaw, Poland

Postep Kardiol Inter 2013; 9, 4 (34): 394-396 DOI: $10.5114 /$ pwki.2013.38873

\begin{abstract}
We report a case of 72-year-old female patient with end-stage chronic kidney disease, undergoing percutaneous coronary intervention $(\mathrm{PCl})$ that resulted in a cardiac arrest caused by a thrombus mediated flow limitation in the left coronary artery. With mechanical cardiopulmonary resuscitation (CPR) PCl of the left main artery was performed successfully during 50 min cardiac arrest. The patient was discharged from the hospital without compromising cardiac function and neurological deficits.
\end{abstract}

Key words: percutaneous coronary intervention, cardiac arrest, cardiopulmonary resuscitation, thrombolitic therapy.

\section{Introduction}

Ischemic complications of percutaneous coronary intervention $(\mathrm{PCl})$ are infrequent but prognostically important. They are strongly associated with subsequent adverse outcomes [1] but fatal $\mathrm{PCl}$ complications are rare and mostly due to left ventricular failure, neurological causes and arrhythmia [2]. Chronic kidney disease (CKD) is associat ed with a greater risk of coronary artery disease (CAD), which is the leading cause of mortality in this population [3]. This high risk group of patients very often requires revascularization [4]. On the other hand, CKD is associated with increased risk of in-hospital mortality as well as adverse ischemic, hemorrhagic, cardiac and cerebrovascular events during treatment with $\mathrm{PCl}$, as compared to patients without CKD [5-7].

\section{Case report}

A 71-year-old woman with end-stage chronic kidney disease (haemodialysis 3 times a week), with a history of non-ST-elevation myocardial infarction (NSTEMI) treated with $\mathrm{PCl}$ of the left main $(\mathrm{LM})$ /left anterior descending (LAD)/circumflex (Cx) artery with two everolimus-eluting stents, with type 2 diabetes, long-standing history of hypertension, and moderate mitral regurgitation, was admitt- ed to the Cardiology Department with a diagnosis of NSTEMI. The echocardiographic examination performed on admission was comparable to the previous study and demonstrated extensive wall motion abnormalities with decreased global ejection fraction $(E F=35 \%)$. The coronary angiography revealed right coronary artery occlusion (as before) and properly functioning stents in the $L M$ and the proximal segments of the LAD and CX. The culprit lesion was a critical narrowing in the medial LAD (Figure 1). As the patient was already receiving aspirin and clopidogrel due to previous myocardial infarction (MI) a decision to perform ad hoc $\mathrm{PCl}$ was taken. Despite initial balloon predilatation of the stenosis (Trek $2.0 \mathrm{~mm} \times 8 \mathrm{~mm}, 10 \mathrm{~atm}$, Abbott Vascular), having good support of the guiding catheter, the stent could not be advanced through the ostium of the LAD. During prolonged manipulations formation of thrombus in the LM, proximal LAD and CX segments (ACT time after heparin bolus $70 \mathrm{U} / \mathrm{kg}$ equal to $267 \mathrm{~s}$ ) occurred (Figure 2). The patient developed cardiogenic shock and subsequently cardiac arrest in the mechanism of pulseless electrical activity (PEA). Immediate manual resuscitation was started and after the patient was intubated a Lund University Cardiac Arrest System (LUCAS) device was engaged to continue automatic chest compression. Despite the administration of intracoronary bolus followed

\section{Corresponding author:}

Marcin Protasiewicz, Department of Cardiology, Medical University of Wroclaw, 213 Borowska St, 50-556 Wroclaw, Poland, tel.: +48 7173642 00, e-mail: mprot@poczta.onet.pl

Received: 19.06.2013, accepted: 10.10.2013 


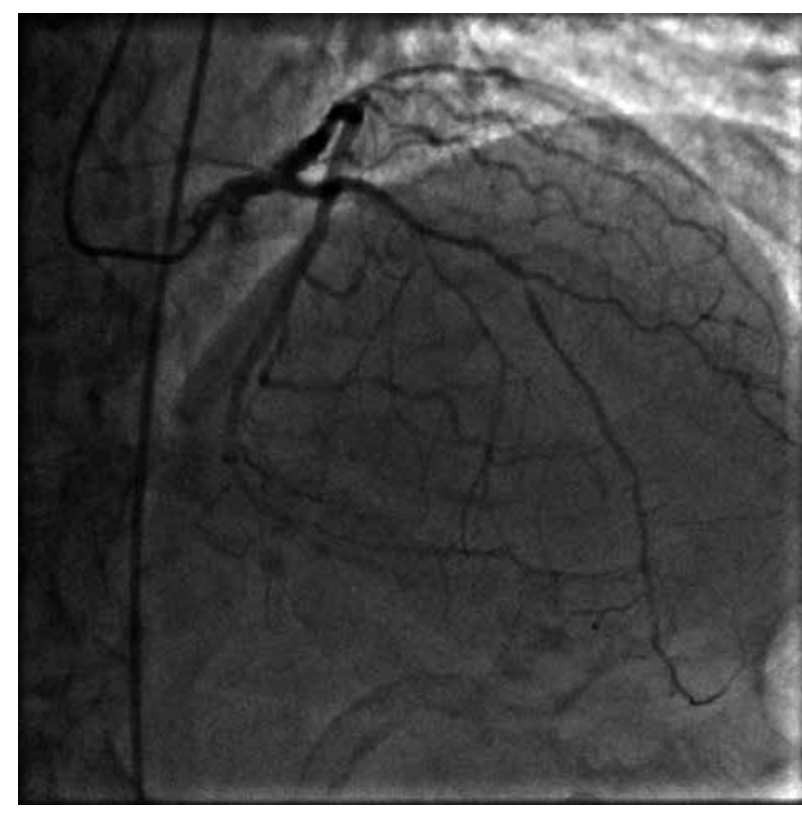

Fig. 1. Angiography of the left coronary artery with properly functioning previously implanted stents

by intravenous infusion of abciximab along with multiple thrombus aspirations with an Export catheter (Medtronic, USA) the coronary angiogram remained unchanged. Spontaneous circulation had not returned. Despite the potential risk of bleeding, $5 \mathrm{mg}$ of intracoronary alteplase was administered. After a few minutes the thrombus began to dissolve but only slight improvement of the flow was observed. Because of the suspicion of coronary artery dissection, a $4.5 \mathrm{~mm} \times 20 \mathrm{~mm}$ stent at 15 atm (Resolute, Medtronic) in the LM/CX was implanted and finally kissing balloon inflation was performed with two $3.0 \mathrm{~mm} \times 20 \mathrm{~mm}$ balloons (Sprinter, Medtronic). After the $\mathrm{PCl}$ spontaneous return of circulation and TIMI-3 flow in the left coronary artery were observed (Figure 3). The whole PCI lasted over 50 min during which ongoing LUCAS support was continuously used. After the procedure the patient with blood pressure of $160 / 80 \mathrm{~mm} \mathrm{Hg}$ and heart rate $110 / \mathrm{min}$ on adrenaline and noradrenaline infusion was transferred to the intensive cardiac unit. Two days later the patient was extubated. Because of the double stent layer in left main coronary artery the patient was subjected to genetic examination of the CYP2C19 gene and light transmission aggregometry (LTA) was performed to assess platelet activity. There was no polymorphism within the CYP2C19 gene but the aggregometry test revealed excessive platelet aggregation of $63 \%$ after stimulation with $5 \mu \mathrm{g}$ of ADP. With this result we decided to change antiplatelet therapy to a more potent platelet inhibitor - ticagrelor. This therapy resulted in proper, $40 \%$, platelet aggregation. The echocardiography examination performed before discharge showed a slight improvement in left ventricular systolic function. No neurological deficits were diagnosed. The patient was discharged from the hospital 10 days later.

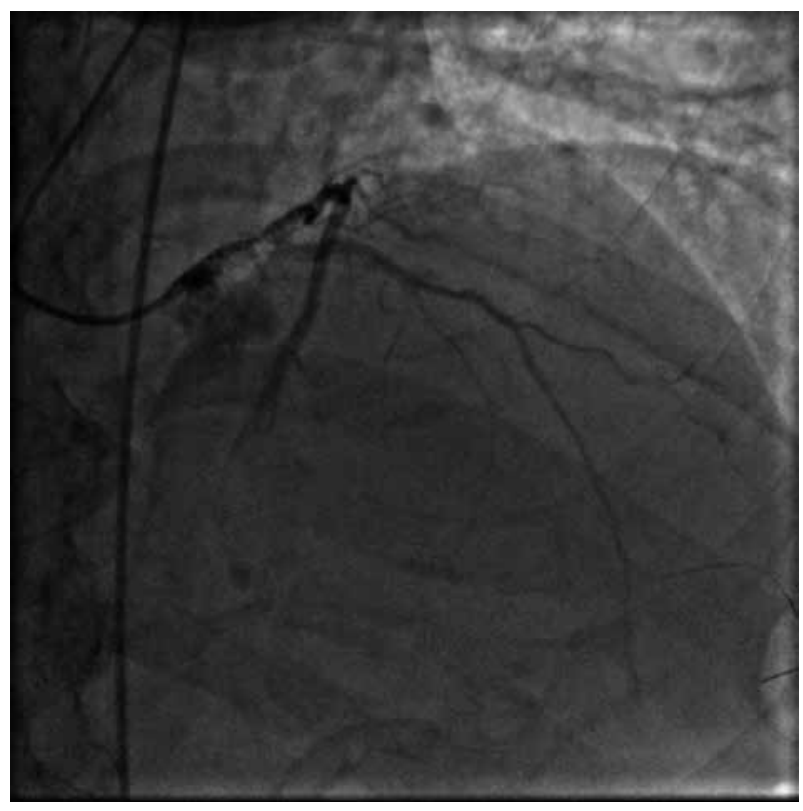

Fig. 2. Thrombus in the left main, proximal left anterior descending and circumflex arteries

\section{Discussion}

The described case shows that a patient at high risk, even with an isolated, simple lesion, can develop very serious complications that should always be reckoned with. The management of cardiac arrest during coronary intervention presents a substantial challenge and effective cardiopulmonary resuscitation with chest compressions is the primary method of circulatory support.

There have been some observations in the past in which continuous mechanical chest compression was

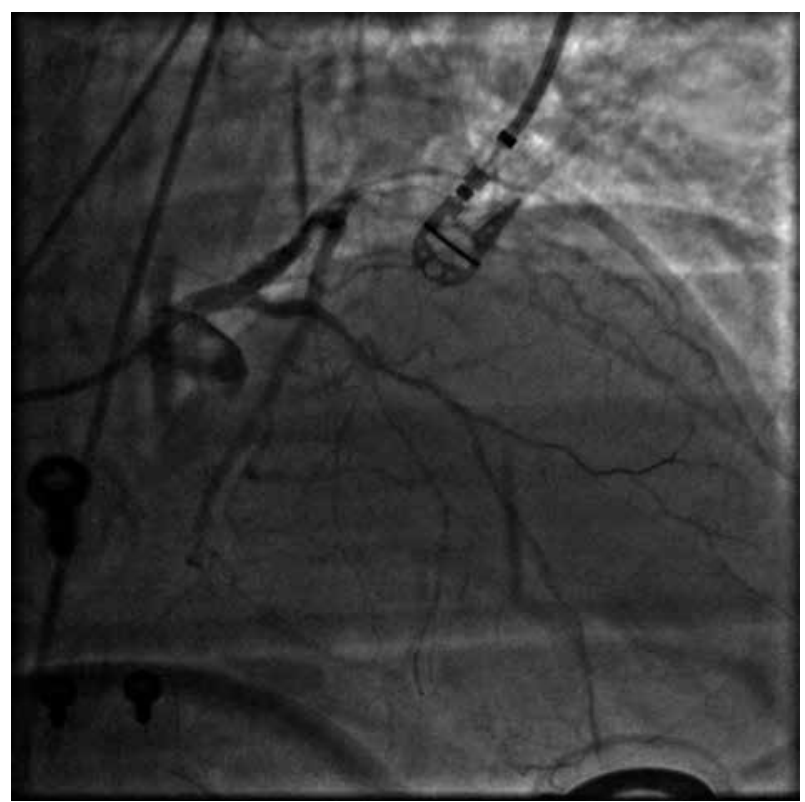

Fig. 3. Restoration of TIMI3 flow in left coronary artery 
used as a bridge to perform a successful $\mathrm{PCl}$ procedure during resuscitation efforts [8-10]. Another aspect of the case described is the use of intracoronary thrombolytic, which, despite the potential complications of bleeding, may be the only effective strategy to deal with a massive intracoronary thrombus [11-13]. Although neither method has been reflected yet in the corresponding guidelines, available publications and the case described above demonstrate the effectiveness of these methods in critical situations.

Finally, an optimal antiplatelet therapy is crucial for successful treatment of ischemic heart events. Chronic kidney disease is one of the reasons for inadequate platelet inhibition with clopidogrel $[14,15]$. We believe that the cause of the serious complication was mechanical but we should remember that high platelet activity is one of the known causes of ischemic complications after $\mathrm{PCl}$ procedures [15]. Prasugrel and ticagrelor, new P2Y12 platelet inhibitors, achieve faster and greater platelet inhibition. In this special, CKD patient, balancing between adequate platelet response and potential bleeding complications, we decided to put our patient on ticagrelor. The drug, in contrast to prasugrel, was shown to reduce major adverse ischaemic events without extensive risk of major bleeding [16]. Fortunately we managed this complication and the patient is alive and not neurologically compromised.

\section{Acknowledgments}

This publication is part of the project "Wrovasc - Integrated Cardiovascular Centre", co-financed by the European Regional Development Fund, within Innovative Economy Operational Program, 2007-2013, implemented in the Provincial Specialized Hospital, Research and Development Center in Wroclaw.

\section{References}

1. McEntegart MB, Kirtane AJ, Cristea E, et al. Intraprocedural thrombotic events during percutaneous coronary intervention in patients with non-ST-segment elevation acute coronary syndromes are associated with adverse outcomes: analysis from the ACUITY (Acute Catheterization and Urgent Intervention Triage Strategy) Trial. J Am Coll Cardiol 2012; 59: 1745-1751.

2. Valle JA, Booher AM, Menees DS, et al. Cause and circumstance of in-hospital mortality among patients undergoing contemporary percutaneous coronary intervention: a root-cause analysis. Circ Cardiovasc Qual Outcomes 2012; 5: 229-235.

3. Kar S, Coats W, Aggarwal K. Percutaneous coronary intervention versus coronary artery bypass graft in chronic kidney disease: Optimal treatment options. Hemodial Int 2011; Suppl. 1: S30-S36.

4. Asim M, Jeffrey RF. Management of acute coronary syndrome in patients with chronic kidney disease: if we don't risk anything, we risk even more. Nephron Clin Pract 2011; 119: c333-c336.

5. Hanna EB, Chen AY, Roe MT, et al. Characteristics and in-hospital outcomes of patients with non-ST-segment elevation myocardial infarction and chronic kidney disease undergoing percutaneous coronary intervention. JACC Cardiovasc Interv 2011; 4: 1002-1008.
6. Saltzman AJ, Stone GW, Claessen BE, et al. Long-term impact of chronic kidney disease in patients with ST-segment elevation myocardial infarction treated with primary percutaneous coronary intervention: the HORIZONS-AMI (Harmonizing Outcomes With Revascularization and Stents in Acute Myocardial Infarction) trial. JACC Cardiovasc Interv 2011; 4: 1011-1019.

7. Kaya E, Cuneo A, Hochadel M, et al. Impact of chronic kidney disease on the prognosis of patients undergoing percutaneous coronary interventions using drug-eluting stents. Clin Res Cardiol 2011; 100: 1103-1109.

8. Azadi N, Niemann JT, Thomas JL. Coronary imaging and intervention during cardiovascular collapse: use of the LUCAS mechanical CPR device in the cardiac catheterization laboratory. J Invasive Cardiol 2012; 24: 79-83.

9. Wagner $\mathrm{H}$, Terkelsen $\mathrm{CJ}$, Friberg $\mathrm{H}$, et al. Cardiac arrest in the catheterisation laboratory: a 5 -year experience of using mechanical chest compressions to facilitate $\mathrm{PCl}$ during prolonged resuscitation efforts. Resuscitation 2012; 81: 383-387.

10. Larsen Al, Hjørnevik AS, Ellingsen CL, et al. Cardiac arrest with continuous mechanical chest compression during percutaneous coronary intervention. A report on the use of the LUCAS device. Resuscitation 2007; 75: 454-459.

11. Higashi $H$, Inaba S, Nishimura K, et al. Usefulness of adjunctive pulse infusion thrombolysis after failed aspiration for massive intracoronary thrombus. Can J Cardiol 2012; 27: 869.e1-2.

12. Kelly RV, Crouch E, Krumnacher H, et al. Safety of adjunctive intracoronary thrombolytic therapy during complex percutaneous coronary intervention: initial experience with intracoronary tenecteplase. Catheter Cardiovasc Interv 2005; 66: 327-332.

13. Triantafyllou K, Metaxopoulos P, Babalis D. Primary percutaneous coronary intervention of an unprotected left main using minicrush drug-eluting stents facilitated by intracoronary reteplase. Catheter Cardiovasc Interv 2011; 77: 515-521.

14. Htun P, Fateh-Moghadam S, Bischofs C, et al. Low responsiveness to clopidogrel increases risk among CKD patients undergoing coronary intervention. J Am Soc Nephrol 2011; 22: 627-633.

15. Morel O, El Ghannudi S, Jesel L, et al. Cardiovascular mortality in chronic kidney disease patients undergoing percutaneous coronary intervention is mainly related to impaired P2Y12 inhibition by clopidogrel. J Am Coll Cardiol 2011; 57: 399-408.

16. James S, Budaj A, Aylward P, et al. Ticagrelor versus clopidogrel in acute coronarysyndromes in relation to renal function: results from the Platelet Inhibition and Patient Outcomes (PLATO) trial. Circulation 2010; 122: 1056-1067. 